\title{
Swallowing disorders in nursing home residents: how can the problem be explained?
}

\author{
This article was published in the following Dove Press journal: \\ Clinical Interventions in Aging \\ 18 February 2013 \\ Number of times this article has been viewed
}

\section{Dália Nogueira' \\ Elizabeth Reis ${ }^{2}$}

'Speech Therapy Department, Escola Superior de Saúde de Alcoitão, Estoril, Portugal; ${ }^{2}$ Department of Quantitative Methods, Lisbon University Institute ISCTE/IUL, Lisbon, Portugal
Correspondence: Dália Maria dos Santos Nogueira

Rua Conde Barão-Alcoitão, 2649-506, Alcabideche, Portugal Email dmsnogueira@gmail.com
Background: The swallowing mechanism changes significantly as people age, even in the absence of chronic diseases. Presbyphagia, a term that refers to aging-related changes in the swallowing mechanism, may be linked to many health conditions and presents itself in distinct ways. Swallowing disorders are also identified as a major problem amongst the elderly population living in nursing homes.

Methods: The study sought to determine the prevalence of swallowing disorders in nursing home residents, to identify the relationship between self-perceived swallowing disorders, cognitive functions, autonomy, and depression, and also to analyze which variables explain the score of the Dysphagia Self-Test (DST). For this purpose, the researchers chose to apply a survey conveying questions on demographic aspects, general health, eating and feeding, as well as instruments to assess functional performance and the 3 ounce Water Swallow Test.

Results: The sample consisted of 272 elderly people living in eight nursing homes in Portugal. Six did not sign the informed consent form. Of the total, $29 \%$ were totally dependent, $33 \%$ were depressed, $45 \%$ had cognitive impairment, and 38\% needed help with feeding. About $43 \%$ of the individuals reported having problems related to eating. Regarding the DST, $40 \%$ showed signs of dysphagia. With respect to the 3 ounce Water Swallow Test, 38\% revealed at least one of the symptoms, wet voice being the most prevalent. Correlation measures showed that age had no linear association with the DST score although correlation with the Barthel Index and Mini Mental State Examination was found to be significant. A linear regression model was estimated with the DST score as the dependent variable and the MMSE and BI scores, gender, age, education, the Geriatric Depression Scale score, 3 ounce Water Swallow Test, and diagnosed conditions (such as neurological disorder, dementia, and cardiorespiratory problems) as explaining variables.

Conclusion: Results showed a high prevalence of dysphagia signs amongst a nursing home population. For the purpose of the present study, both a subjective and an objective assessment were applied. Results pointed to a significant statistical relation between objective and subjective measures, thus indicating that a self-perception test should be included in the assessment of swallowing disorders in a nursing home population. Notwithstanding, it should not be used as a single or principal measure as it is influenced by the individuals' cognitive condition.

Keywords: deglutition, deglutition disorders, elderly

\section{Introduction}

An understanding of normal swallowing is essential to identify swallowing disorders in older people. It is still unclear which impairments are related to primary aging and which are the consequence of diseases. ${ }^{1,2}$ Aging effects on human deglutition have been found in several studies and are known to affect both the oral and pharyngeal phases 
of swallowing. ${ }^{3}$ As people age, the cortical hemispheric control of swallowing develops compensatory mechanisms commonly seen in other sensorimotor functions. The study of Malandraki et $\mathrm{al}^{3}$ revealed that areas involved in swallowing processes showed limited activity in older people. Other studies have found that healthy older people demonstrated decreased intraoral pressure, increased multiple swallows, and increased frequency of inspiration and coughing during swallow. ${ }^{1}$ According to McKee et $\mathrm{al}^{4}$ and Nicosia et $\mathrm{al},{ }^{5}$ aging is associated with a slowing of the swallow response in the pharynx, due to both central and peripheral factors, and this change may have an impact on the bolus drive. Overall, tongue pressure declines with age, and the implications of these changes result in further efforts to produce swallowing pressures; ${ }^{6}$ however, the required high activity level of tongue muscles may play a role in their movement preservation when other skeletal muscles are more likely to show functional effects of such changes. ${ }^{7}$ The study by Higashijima ${ }^{8}$ points out that the swallowing function in the aged is also influenced by bolus size.

Presbyphagia, a term that refers to aging-related changes in the swallowing mechanism, may present in several ways: as the lack of muscle strength, complicating the bolus propulsion; the diminished lingual pressure, obstructing bolus driving; halting of the bolus whilst swallowing, leading to a more difficult cleansing of residues; the decline of taste and smell, making it more difficult to initiate swallowing; difficulty in controlling bolus from the anticipatory phase; the entering of the bolus into the lower airway; and finally, the lack of teeth and the wearing, or not, of complete dentures, which influence chewing. The disruption of the capacity to swallow in older adults has long-range consequences with severe health implications. ${ }^{9}$ It may result in dehydration, malnutrition, airway obstruction, and aspiration pneumonia. ${ }^{10}$ Advancing age is also increasingly associated with comorbid situations, predisposing individuals to frailty that, in the event of an aspiration episode, may give rise to increased morbidity and mortality. ${ }^{11}$ Presbyphagia becomes clinically more relevant from the age of 80 onwards, and frail older people with dysphagia present higher mortality rates. ${ }^{12}$ According to Cabre et al, ${ }^{13}$ oropharyngeal dysphagia is a highly prevalent clinical finding in older people with pneumonia. Moreover, the repercussions of dysphagia are both physical and also emotional and social, leading to depression and social isolation, with a great impact on the quality of life.

Cognitive and perceptual changes are mostly present in older people, constituting an important risk factor in swallowing disorders. ${ }^{14}$ Another important but often disregarded issue can be the elderly person's self-perception of his/her health status and sense of loss of well-being, which is normally associated with swallowing and eating impairments. ${ }^{15}$ A study conducted by Holland et a ${ }^{16}$ showed that dysphagia was correlated with age and depression. However, there was no significant correlation with memory, recall, or mental performance.

There is a relatively high prevalence of dysphagia in the community-based geriatric population and a significant quality of life impairment related to eating and feeding disorders. ${ }^{17}$ This same problem is even more prevalent in nursing homes. Up to $40 \%$ of people in permanent-care settings are dysphagic, ${ }^{9}$ and between $50 \%$ to $75 \%$ of nursing home residents have some difficulty in swallowing. ${ }^{18}$ A study by Wilkinson and de Picciotto ${ }^{19}$ investigated the prevalence of subjectively reported swallowing problems, in a group of retirement village residents. Results revealed a prevalence of $44 \%$ of subjects who experienced swallowing problems that interfered with their day living functioning. Further, dementia is a major problem among older people living in nursing homes; Alzheimer's is also the most common at-risk condition, as it is the cause of a loss of the conscious part of mastication and nonreflex swallowing. ${ }^{20}$ In nursing care settings, individuals are often seen choking while they are eating and drinking. This situation not only poses difficulties for professionals but also interferes with the quality of life of the individuals and their families. ${ }^{21}$ Community-acquired pneumonia is another major cause of morbidity and mortality in the elderly and is the leading cause of death amongst nursing home residents. Aspiration is an important etiologic factor leading to pneumonia. ${ }^{22}$ Dysphagia can also be caused by many medical conditions commonly affecting older people as well as by the effects of medication. ${ }^{23}$

To be effective, dysphagia management requires a multidisciplinary team approach. Older people who are at risk should be evaluated in different ways, as some risks are caused by an acute condition and others by chronic disease or handicap. Management of dysphagia in nursing homes is sometimes curative but more often adaptive and/or palliative and should not be restricted to meals, and it starts with changing some decubitus and head and trunk positions. Nevertheless, detecting older people at risk, through an accurate, specialized clinical and functional diagnosis, is mandatory. ${ }^{20}$

This study intended to answer the research question: What is the nature of swallowing and eating impairment in a community of nursing home residents? 
The study aimed to (1) determine the prevalence of swallowing and feeding disorders in a sample of nursing home residents; (2) identify the relationship between selfperceived swallowing disorders and cognitive and functional performance; and (3) identify the variables that best explain self-perceived swallowing disorders.

\section{Methods}

This is a cross-sectional, descriptive, and correlational study based on a sample of 272 senior residents of eight nursing homes. Six persons in the sample did not sign the informed consent. After the Nursing Home's Ethics Committee approval, data were collected during face-to-face interviews with residents, and conducted by previously trained researchers. All the residents at the eight nursing homes were initially eligible to participate, since no exclusion criteria were applied apart from the level of alertness, cooperation, and ability to comprehend that were necessary to complete the tests. When individuals were incapable of responding or when the information could not be collected, test items were marked as missing values. Individuals were excluded from each test after a preliminary approach by the interviewer (the Tables show the percentage of individuals evaluated in each test). In order to identify swallowing disorders, a combination of different instruments was applied to summarize crucial assessment domains. Six instruments were employed to characterize the elderly swallow profile: (1) a generic survey giving information about the demographic profile, diseases, and comorbid conditions as well as functional (motor and cognitive) performance; (2) the Barthel Index (BI) was used to assess activities of daily living; (3) the Mini Mental State Examination (MMSE) was applied to evaluate cognitive impairment; (4) swallow and eating profiles were assessed by a Speech and Language therapist, using the 3 ounce Water Swallow Test (3OZwst); (5) the Dysphagia Self-Test (DST) questionnaire was administered to the residents who were able to respond; (6) The Geriatric Depression Scale (GDS) was used to assess depression symptoms. ${ }^{24-29}$

The BI is an ordinal scale used to measure performance in activities of daily living and is scored from 0 to 100 . The lowest value means total dependency, and the highest value reveals total independence.

The MMSE is a 30 -item questionnaire used to screen cognitive impairment. ${ }^{25}$ It ranges from 0 to 30 . The lowest value indicates total cognitive impairment, and the highest indicates no signs of cognitive impairment (values are scored in accordance with education levels). The GDS was first created by Yesavage ${ }^{26}$ and has been tested and used extensively with older populations. Of the 15 items, the presence of ten indicates depression. The DST ${ }^{27,28}$ is a questionnaire with ten items that relies on self-assessment of symptoms and can help determine whether the individual feels that he/she is at risk of dysphagia/aspiration. The score ranges from 0 (no symptoms) to 10 (all symptoms). In accordance with previous literature, ${ }^{27}$ one point was added for all the DST items that can reveal risk of penetration and/or aspiration. The 3OZwst, created by DePippo et al, ${ }^{29}$ is a sensitive screening tool to identify patients at risk. Individuals are required to drink 3 ounces of water without interruption. Those who stop, cough, choke, or show a wet-hoarse vocal quality during the test or for 1 minute afterwards are considered to have failed.

Statistical analysis included frequency distributions and descriptive measures, correlation coefficients, hypothesis tests for equality of means, and multiple regression modeling. As well, $t$-tests for equality of means were applied to compare two independent groups (males and females); in the presence of more than two groups (four education levels), one-way analysis of variance (ANOVA) and multiple comparison tests were chosen to compare means. Linear Regression was applied to estimate the coefficients of a linear equation, involving one or more independent variables that best predicted the value of the DST score (the dependent variable). Linear regression modeling assumes that the dependent and independent variables are quantitative. Categorical variables, such as gender and education need to be recoded to binary (dummy) variables. When a regression model is estimated, reality is simplified, and all the independent variables not included in the model are included in the random error (which can also be the result of measuring error). Further assumptions of the multiple linear regression models included the existence of linearity on the relationship between the dependent and the independent variables, normality of the random error with zero mean and constant variance, no correlation between the random error and the independent variables, and no collinearity between independent variables.

Stepwise estimation was used. At each step, the independent variable not in the equation with highest significance was included, if that probability was sufficiently high. Variables already in the regression equation were removed if their significance ceased to be high enough. The method terminated when no more variables were eligible for inclusion or removal.

If the data appeared to violate one or more assumptions (such as normality or constant variance), the alternative became the generalized linear model, which expanded the 
general linear model, allowing the dependent variable to have a non-normal distribution and to linearly relate to the factors and covariates via a specified link function.

\section{Results}

The sample included 266 (six did not sign the informed consent) residents, with an average age of $82 \pm 10$ years. Of these, $75 \%$ were females, $48 \%$ had no formal education, $41 \%$ were single, and $41 \%$ were widowers. About $68 \%$ of the residents had more than one diagnosis identified. The most frequent disease was cardiopulmonary problems, followed by osteoarticular disease, dementia, and psychiatric diseases.

The results of the BI showed that $86.6 \%$ of the residents had some level of dependency, and $28.7 \%$ were totally dependent (Table 1). Residents showing some type of cognitive impairment totaled $44.9 \%$. The results for the GDS indicated that $30.9 \%$ of the sample had symptoms of depression.

According to the BI item that assessed independence for feeding, almost half of the residents were in need of assistance. Nevertheless, $80.5 \%$ ate normal food (Table 2); regarding textures, the greatest difficulty was felt when eating solids $(22.8 \%)$.

One point was added for all the DST items that revealed a risk of penetration and/or aspiration (Table 3). When the threshold "seven or more difficulties" was used, signs of dysphagia were present in $40.1 \%$ of the residents. The 3 OZwst and the deviation from normal swallowing was also registered, namely the presence of cough, wet voice, or

Table I Scores for the BI, the MMSE, and the GDS

\begin{tabular}{lll}
\hline & Frequency & Percent \\
\hline Barthel index & 78 & 28.7 \\
Total dependency & 39 & 14.3 \\
Severe dependency & 41 & 15.1 \\
Moderate dependency & 34 & 12.5 \\
Mild dependency & 44 & 16.2 \\
Minimal dependency & 24 & 8.8 \\
Independent & 260 & 95.6 \\
Total & 6 & \\
Missing & & 44.9 \\
Mini Mental State examination & 122 & 43.8 \\
Cognitive impairment & 119 & 88,6 \\
No cognitive impairment & 241 & \\
Total & 15 & 22.4 \\
Missing & & 30.9 \\
Geriatric depression scale & 61 & 53.3 \\
Without depression signs & 84 & \\
With depression signs & 145 & \\
Total & 121 & \\
Missing & Min Menta State Exam & \\
\hline Abreviations: BI, Bathe & & \\
\hline
\end{tabular}

Abbreviations: BI, Barthel Index; MMSE, Mini Mental State Examination; GDS, Geriatric Depression Scale.
Table 2 Barthel Index (independence for feeding) and feeding profile

\begin{tabular}{lll}
\hline & Frequency & $\%$ \\
\hline Barthel Index feeding levels & & \\
Total dependency & 24 & 8.8 \\
Total assistance & 26 & 9.6 \\
Some assistance & 30 & 11.0 \\
Minimal assistance & $5 \mathrm{I}$ & 18.8 \\
Independency & 114 & 41.9 \\
Form of feeding & & \\
Normal & 219 & 80.5 \\
NG tube & 2 & 0.7 \\
PEG tube & 1 & 0.4 \\
Puréed food & 33 & 12.1 \\
Thickener & $\mathrm{I}$ & 0.4 \\
Syringe & 2 & 0.7 \\
NG tube for liquids & $\mathrm{I}$ & 0.4 \\
\hline
\end{tabular}

Abbreviations: NG, nasogastric; PEG, percutaneous endoscopic gastrostomy.

both, to obtain a more objective evaluation from a Speech and Language Pathologist: it was found that $38.2 \%$ of the residents had problems, of which "wet voice" was the most prevalent (Table 3).

The mean score of the DST was compared by gender and level of education. No significant differences were found between males and females, but a higher education level was found to have a positive effect on the DST. The Pearson correlation coefficient was used to identify the degree of linear association between the DST and age, the BI, the MMSE, and the BI feeding indicator. All correlations but one were significant, though not strong: $\operatorname{MMSE}\left(\mathrm{r}_{(272)}=-0.221\right.$, $P<0.001)$; $\mathrm{BI}\left(\mathrm{r}_{(272)}=-0.268, P<0.001\right)$; BI feeding $\left(\mathrm{r}_{(272)}=-0.268, P<0.001\right)$; the only exception was the correlation with age, which was not significant $\left(\mathrm{r}_{(272)}=0.091\right.$, $P=0.162)$ (Table 4).

ANOVA was applied to compare the DST mean scores between the groups defined by the self-perception of health status. The results showed significant mean differences. The group of residents with a poor self-perceived health status had the highest mean scores of DST (worst swallowing) $\left(\mathrm{F}_{(2,158)}=5.859, P=0.004\right)$.

To identify the variables that best explain DST variations, a regression model was estimated using the DST as the dependent variable and the MMSE and BI scores, gender, age, education, the GDS Score, 3OZwst, and predominant diseases as independent variables. The final adjusted model was significant and explained $47.2 \%$ of the DST score variation $\left(\mathrm{F}_{(7,115)}\right.$, adjusted $\left.\mathrm{R}^{2}=0.472, P<0.001\right)$. Age, education, the $3 \mathrm{OZwst}$, the MMSE, and GDS scores and also cardiorespiratory problems had significant effects $(P<0.05)$ on the DST score (Table 5). Education had a negative effect 
Table 3 DST and the 3OZwst

\begin{tabular}{|c|c|c|c|}
\hline \multirow[t]{2}{*}{ DST Items } & \multirow[t]{2}{*}{ Weight } & \multicolumn{2}{|l|}{ Yes } \\
\hline & & $\mathbf{N}$ & $\%$ \\
\hline I. Does food sometimes go down the wrong pipe? & 1 & 15 & 6 \\
\hline 2. Does your voice sometimes sound "gurgly" or wet when you eat? & 2 & 34 & 15 \\
\hline 3. Is eating sometimes less enjoyable than it used to be? & I & 86 & 37 \\
\hline 4. Do you sometimes have trouble clearing food from your mouth in one swallow? & I & 109 & 47 \\
\hline 5. Do you sometimes get the feeling that food is stuck in your throat? & 2 & 48 & 21 \\
\hline 6. Have you had repeated pneumonia or other respiratory illnesses? & 2 & 56 & 24 \\
\hline 7. Have you ever lost weight without trying? & 2 & 75 & 32 \\
\hline 8. Do you often have trouble swallowing medications? & 2 & 53 & 23 \\
\hline 9. Do you often choke or cough when you eat solid foods or swallow liquids? & 2 & 114 & 49 \\
\hline 10. Do you often have difficulty swallowing a specific food or liquid? & 2 & 43 & 18 \\
\hline \multicolumn{4}{|l|}{ DST } \\
\hline Less or equal to 6 & & & 44.1 \\
\hline 7 or more & & & 40.1 \\
\hline \multirow[t]{2}{*}{ Missing } & & & 15.8 \\
\hline & $\mathbf{N}$ & $\%$ & Valid \% \\
\hline \multicolumn{4}{|l|}{$30 Z$ wst } \\
\hline No problem & 170 & 62.5 & 72.6 \\
\hline Cough & 21 & 7.7 & 9 \\
\hline Wet voice & 24 & 8.8 & 10.3 \\
\hline Both & 19 & 7 & 8.1 \\
\hline
\end{tabular}

Abbreviations: DST, Dysphagia Self-Test; 3OZwst, 3 ounce Water Swallow Test.

on the DST score ie, residents with lower levels of education had higher levels of self-perceived swallowing disorders. DST scores increased with age, with higher GDS scores, cardiorespiratory problems, and decreased in those with no risk detected by the $3 \mathrm{OZ}$ swt.

However, a generalized linear model was also estimated to validate the previous results, due to the violation of some of the linear regression model assumptions (normality and homoscedasticity of residuals). The dependent variable was set to have a Poisson distribution (since the dependent variable has non-negative integer values, can be thought of as the number of occurrences of an event, and shows positive skewness). The log link function was appropriate for positive skewed distributions.

The results confirm the significant effect of education, age, the GDS score, and cardiorespiratory problems on the level of DST, but the MMSE index became nonsignificant and was excluded from the final model (Table 6). The DST score was found to be higher for those with no education and

Table 4 Correlations between DST, and BI, MMSE, BI (feeding)

\begin{tabular}{ll}
\hline DST/BI & $r=-0.268, P$-value $<0.00$ I \\
DST/MMSE & $r=0.22 I, P$-value $<0.00$ I \\
DST/BI (feeding) & $r=0.268, P$-value $<0.00$ I
\end{tabular}

Abbreviations: DST, Dysphagia Self-Test; BI, Barthel Index; MMSE, Mini Mental State Examination. lower for those with 4 years education, when compared with the group of residents with more than 4 years of education; lower for those with no problems in the 3OZwst when compared with those with both wet voice and cough; increased when age and GDS scores increased; and was lower for those with cardiorespiratory diseases (Table 7).

\section{Discussion}

The study sought to determine the prevalence of swallowing disorders in nursing home residents and to identify the relationship between the DST score and a set of health and sociodemographic variables, cognitive functions, performance of activities of daily living, and depression.

Clinicians need to recognize the heterogeneity of health determinants and how these factors may influence approaches to dysphagia in different care settings. Current economic restraints are putting pressure on nursing home facilities to identify key issues that are associated with various outcomes, including the burden of respiratory infection resulting from aspiration. Neither the scope of the problem nor the prevalence of dependence and cognitive impairment in the elderly that were reported in this study surprised the authors. The functional profile identified in the present study points to higher prevalence of functional dependence in females, with cognitive impairment, and in the presence of signs of 
Table 5 Linear regression model to explain the variation of the DST score

\begin{tabular}{|c|c|c|c|c|c|}
\hline & \multicolumn{2}{|c|}{ Unstandardized coefficients } & \multirow{2}{*}{$\begin{array}{l}\text { Standardized coefficients } \\
\text { Beta }\end{array}$} & \multirow[t]{2}{*}{$t$} & \multirow[t]{2}{*}{$P$-value } \\
\hline & B & SE & & & \\
\hline (Constant) & -9.005 & 3.134 & & -2.873 & 0.005 \\
\hline Age & 0.154 & 0.030 & 0.363 & 5.147 & 0.000 \\
\hline $\begin{array}{l}\text { No formal } \\
\text { education* }\end{array}$ & 2.530 & 0.726 & 0.273 & 3.487 & 0.001 \\
\hline $3 O Z w s t=n_{n} e^{\S}$ & -2.729 & 0.591 & -0.310 & -4.615 & 0.000 \\
\hline GDS score & 0.300 & 0.073 & 0.279 & 4.113 & 0.000 \\
\hline 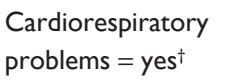 & 1.257 & 0.532 & 0.159 & 2.362 & 0.020 \\
\hline 4 years education* & -1.414 & 0.611 & -0.178 & -2.313 & 0.023 \\
\hline MMSE & 0.109 & 0.052 & 0.147 & 2.084 & 0.039 \\
\hline
\end{tabular}

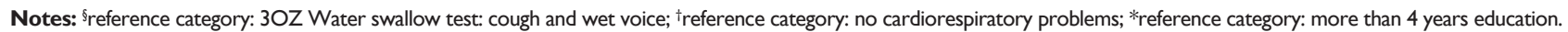
Abbreviations: DST, Dysphagia Self-Test; 3OZwst, 3 ounce Water Swallow Test; GDS, Geriatric Depression Scale; MMSE, Mini Mental State Examination; B, regression coefficient; SE, standard error.

depression or cognitive disorders. According to reported problems pointing to risk of dysphagia, about $40 \%$ of nursing home residents showed symptoms, as was similarly reported in the study of Humbert et al. ${ }^{9}$ The variables that best explain the total score of the DST were similar in both classic and generalized regression models. Education levels that traditionally explain self-perceived health status also showed a high correlation with DST. Residents with a higher cognitive performance showed higher scores for the DST, which may indicate a greater awareness of the symptoms. The GDS score explains the greater difficulties reported in the DST and may be related to poor well being, which is in line with Feinberg's opinion; ${ }^{15}$ it may also be due to the consumption of medication, but this variable was not introduced in the present study, due to the lack of information. The use of medication should be included as an explanatory variable, since many medical conditions and the use of medication can cause dysphagia. ${ }^{23}$

According to the literature review, ${ }^{16}$ cognitive factors, such as memory recall, do not seem to influence dysphagia symptoms. On the other hand, depression is associated with dysphagia, which suggests a potential interaction effect. This could be linked to associations with quality of life or psychological factors. The 3OZwst also explains the DST

Table 6 Generalized linear model - tests of model effects

\begin{tabular}{lll}
\hline & Wald chi-square & P-value \\
\hline (Intercept) & 0.123 & 0.726 \\
Education & 34.050 & 0.000 \\
Test 3OZwst & 40.540 & 0.000 \\
Cardiorespiratory problems & 5.779 & 0.016 \\
Age & 27.431 & 0.000 \\
GDS score & 19.925 & 0.000 \\
\hline
\end{tabular}

Abbreviations: 3OZwst, 3 ounce Water Swallow Test; GDS, Geriatric Depression Scale. score, indicating that the $3 \mathrm{OZ}$ wst can be a simple and easily applied method for obtaining basic information about swallow functions, ${ }^{29}$ even if not as a single measure.

Our own experience also showed that a more objective assessment should include the caregiver's opinion. The DST applied to the elderly is influenced by many subjective factors, including each individual's ability to interpret his/her own symptoms. As many nursing home residents showed a substantial cognitive decline, the DST should be used only as an indicator and not as an objective evaluation instrument. This type of tool should be complementary, and the results should be regarded with caution especially in a population with high levels of cognitive impairment. Symptoms may, in many cases, be undervalued, putting the elderly at risk. However, it is important to include a subjective assessment of dysphagia symptoms, since the ability to perform a given function is closely linked to the individual's perception of his/her own difficulties.

Table 7 Generalized linear model - parameter estimates

\begin{tabular}{llll}
\hline & B & Wald chi-square & $P$-value \\
\hline (Intercept) & 0.302 & 0.633 & 0.426 \\
No education* & 0.231 & 4.954 & 0.026 \\
Knows how to read & -0.067 & 0.308 & 0.579 \\
and write* & & & \\
4 years education* & -0.234 & 5.652 & 0.017 \\
3OZwst = none & -0.410 & 15.677 & 0.000 \\
3OZwst = cough & 0.049 & 0.124 & 0.725 \\
3OZwst = wet voice & 0.027 & 0.046 & 0.830 \\
Cardiorespiratory & -0.161 & 5.779 & 0.016 \\
problems = no & & & \\
Age & 0.021 & 27.431 & 0.000 \\
GDS & 0.039 & 19.925 & 0.000 \\
\hline
\end{tabular}

Notes: *reference category: more than 4 years education; ${ }^{\text {rreference category: }}$ 3OZwst: both; †reference category: yes.

Abbreviations: 3OZwst, 3 ounce Water Swallow Test; GDS, Geriatric Depression Scale; B, regression coefficient. 
Further development of specific screening tools for older people is necessary to efficiently and objectively identify problems and provide early intervention in nursing homes, where dysphagia is responsible for many acute and chronic respiratory diseases, increasing the state of dependency, and contributing to rates of morbidity and mortality.

\section{Disclosure}

The authors report no conflicts of interest in this work.

\section{References}

1. Nilsson H, Ekberg O, Olsson R, Hindfelt B. Quantitative aspects of swallowing in an elderly nondysphagic population. Dysphagia. 1996; 11(3):180-184.

2. Sura L, Madhavan A, Carnaby G, Crary MA. Dysphagia in the elderly: management and nutritional considerations. Clin Interv Aging. 2012; 287-298.

3. Malandraki GA, Perlman AL, Karampinos DC, Sutton BP. Reduced somatosensory activations in swallowing with age. Hum Brain Mapp. 2011;32(5):730-743.

4. McKee GJ, Johnston BT, McBride GB, Primrose WJ. Does age or sex affect pharyngeal swallowing? Clin Otolaryngol Allied Sci. 1998; 23(2):100-106.

5. Nicosia MA, Hind JA, Roecker EB, et al. Age effects on the temporal evolution of isometric and swallowing pressure. J Gerontol A Biol Sci Med Sci. 2000;55(11):M634-M640.

6. Steele CM, Van Lieshout P. Tongue movements during water swallowing in healthy young and older adults. J Speech Lang Hear Res. 2009;52(5):1255-1267.

7. Bennett JW, van Lieshout PH, Steele CM. Tongue control for speech and swallowing in healthy younger and older subjects. Int J Orofacial Myology. 2007;33:5-18.

8. Higashijima M. Influence of age and bolus size on swallowing function: basic data and assessment method for care and preventive rehabilitation. Am J Occup Ther. 2010;64(1):88-94.

9. Humbert IA, Robbins J. Dysphagia in the elderly. Phys Med Rehabil Clin NAm. 2008;19(4):853-866.

10. Allepaerts S, Delcourt S, Petermans J. Swallowing disorders in the elderly: an underestimated problem. Rev Med Liege. 2008;63(12): 715-721. French.

11. Hudson HM, Daubert CR, Mills RH. The interdependency of proteinenergy malnutrition, aging, and dysphagia. Dysphagia. 2000;15(1): $31-38$.
12. Rofes L, Arreola V, Romea M, et al. Pathophysiology of oropharyngeal dysphagia in the frail elderly. Neurogastroenterol Motil. 2010; 22(8):851-858.

13. Cabre M, Serra-Prat M, Palomera E, Almirall J, Pallares R, Clavé P. Prevalence and prognostic implications of dysphagia in elderly patients with pneumonia. Age Ageing. 2010;39(1):39-45.

14. Elliott JL. Swallowing disorders in the elderly: a guide to diagnosis and treatment. Geriatrics. 1988;43(1):95-100, 104, 113.

15. Feinberg MJ. Swallowing versus eating impairment in nursing home residents. Dysphagia. 1997;12(1):51.

16. Holland G, Jayasekeran V, Pendleton N, Horan M, Jones M, Hamdy S. Prevalence and symptom profiling of oropharyngeal dysphagia in a community dwelling of an elderly population: a selfreporting questionnaire survey. Dis Esophagus. 2011;24(7):476-480.

17. Chen PH, Golub JS, Hapner ER, Johns MM 3rd. Prevalence of perceived dysphagia and quality-of-life impairment in a geriatric population. Dysphagia. 2009;24(1):1-6.

18. O'Loughlin G, Shanley C. Swallowing problems in the nursing home: a novel training response. Dysphagia. 1998;13(3):172-183.

19. Wilkinson T, de Picciotto J. Swallowing problems in the normal ageing population. S Afr J Commun Disord. 1999;46:55-64.

20. Rumeau P, Vellas B. Dysphagia, a geriatric point of view. Rev Laryngol Otol Rhinol (Bord). 2003;124(5):331-333. French.

21. Kyle G. Managing dysphagia in older people with dementia. $B r J$ Community Nurs. 2011;16(1):6-10.

22. Marik PE, Kaplan D. Aspiration pneumonia and dysphagia in the elderly. Chest. 2003;124(1):328-336.

23. Logemann JA. Effects of aging on the swallowing mechanism. Otolaryngol Clin North Am. 1990;23(6):1045-1056.

24. Mahoney FI, Barthel DW. Functional evaluation: the Barthel Index. Md State Med J. 1965;14:61-65.

25. Guerreiro M, Silva A, Botelho M. Adaptação à população portuguesa na tradução do Mini Mental State Examination (MMSE). [Translation and adaptation of the MMSE to the Portuguese population]. Revista Portuguesa de Neurologia. 1994;1:9-10. Portuguese.

26. Yesavage JA. Geriatric Depression Scale. Psychopharmacol Bull. 1988;24(4):709-711.

27. Logemann JA, Stewart C, Hurd J, et al. Dysphagia Care Among Seniors in Los Angeles County: Planning and Pilot Study. Presented at the California Speech-Hearing-Language Association Convention, Monterey, Calfornia: 2008 April.

28. Baker BM, Fraser AM, Baker CD. Long-term postoperative dysphagia in oral/pharyngeal surgery patients: subjects' perceptions vs videofluoroscopic observations. Dysphagia. 1991;6(1):11-16.

29. DePippo KL, Holas MA, Reding MJ. Validation of the 3-oz water swallow test for aspiration following stroke. Arch Neurol. 1992; 49(12):1259-1261.
Clinical Interventions in Aging

\section{Publish your work in this journal}

Clinical Interventions in Aging is an international, peer-reviewed journal focusing on evidence-based reports on the value or lack thereof of treatments intended to prevent or delay the onset of maladaptive correlates of aging in human beings. This journal is indexed on PubMed Central, MedLine, the American Chemical Society's 'Chemical Abstracts

\section{Dovepress}

Service' (CAS), Scopus and the Elsevier Bibliographic databases. The manuscript management system is completely online and includes a very quick and fair peer-review system, which is all easy to use. Visit http://www.dovepress.com/testimonials.php to read real quotes from published authors. 